\title{
Direct Coombs Test Positivity in B-Chronic Lymphoid Leukemia: a Marker of Advanced Clinical Disease
}

\author{
Syeda Alia Abbas*, Rozina Zeeshan, Sadia Sultan, Syed Mohammad Irfan
}

\begin{abstract}
Background: Chronic lymphoid leukemia (CLL) is a malignant hematopoietic disorder, the most common of all adult leukemias with a distinctive immunophenotype. It is well established that CLL patients can have autoimmune complications, amongst them autoimmune hemolytic anemia as the most frequent. This study was carried out to determine the frequency of direct Coombs Test positivity in CLL patients and its possible correlation with Rai staging, hematological parameters and biochemical markers. Materials and Methods: This descriptive cross sectional study was carried at Liaquat National Hospital from January 2011 to June 2013. Sixty untreated patients with B- chronic lymphoid leukemia were enrolled. Complete blood count, direct Coombs test, serum urea, creatinine, uric acid and LDH levels were determined. Data were compiled and analyzed using SPSS version 21. Results: Out of 60 patients, $42(70 \%)$ were males and $18(30 \%)$ were females. Mean age was $59 \pm 9.2$ years. Male to female ratio was 2.1: 1 . The frequency of direct antiglobulin test (DAT) positivity was found to be $23.3 \%$. The monospecific IgG was positive in 11 patients $(18.3 \%)$; C3d positivity was evident in 1 patient $(1.6 \%)$ and 2 patients $(3.3 \%)$ had dual IgG and C3d positivity. The mean hemoglobin was $10.8 \pm 2.4 \mathrm{gm} /$ dl. Significantly low mean hemoglobin of $8.3 \pm 3.0 \mathrm{gm} / \mathrm{dl}$ was seen in Coombs positive patients compared with negative patients having a mean hemoglobin level of $11.7 \pm 1.6 \mathrm{gm} / \mathrm{dl}(\mathbf{P}<0.001)$. DAT positivity also demonstrated a positive association with advanced Rai stage III disease $(P<0.01)$. No associations were noted with age, gender and biochemical markers. Conclusions: Direct Coombs test positivity in CLL in our patients, unlike in Western studies, appears relatively high, indicating significant autoimmune hemolytic anemia and advanced Rai stage in our setting. DAT positivity can be considered as a surrogative marker for advanced clinical disease.
\end{abstract}

Keywords: B-chronic lymphoid leukemia - direct Coombs test - autoimmune hemolytic anemia

Asian Pac J Cancer Prev, 16 (14), 6007-6010

\section{Introduction}

Chronic lymphoid leukemia is the most common type of adult leukemia in the western countries with an incidence of about 5 cases per 100,000 residents annually (Rozman et al., 1995; Zwiebel et al., 1998; Xu et al., 2009). However, it is infrequent in the Eastern countries (Xu et al., 2009). The disease affects mainly people of advanced age, but about $20 \%$ of patients are younger than 55 years with the predominance of male gender (Mauro et al., 1999; Zeeshan et al., 2015).

The disease is characterized by a clonal accumulation of mature apoptosis-resistant neoplastic cells with a distinctive immunophenotype (i.e. SmIgdim, CD5+, CD19+, CD20dim, CD23+) in peripheral blood, bone marrow, lymph nodes and other lymphoid tissues (Hodgson et al., 2011; Shahjahani et al., 2015). It is a heterogeneous disease with a variable clinical outcome (Mozaheb et al., 2012; Zeeshan et al., 2015).

CLL is frequently associated with autoimmune complications (Polliack et al., 1992). Three frequently associated autoimmune hematological complications includes autoimmune hemolytic anemia (AIHA), idiopathic thrombocytopenic purpura (ITP) and pure red cell aplasia (Diehl et al., 1998). The most frequently occurring autoimmune complication in CLL is AIHA (Engelfriet et al., 1992). It occurs in $10 \%$ to $25 \%$ of patients during their disease course (Dearden et al., 2008). Previous studies have shown that AIHA is usually observed in advanced stages of the disease and that CLL patients with AIHA belong to a poor prognostic group (De Rossi et al., 1988; Foon et al., 1990).Determination of patient outcome with advanced stage CLL on the basis of the origin of the cytopenias (immune versus infiltrative) can be important because of prognostic and treatment considerations (Moreno et al., 2008).

Cytopenias due to immune disorder as in AIHA could be sought out with DAT positivity with unexplained anemia (Ehsan et al., 2013). DAT positivity leads to reduced overall survival; hence DAT status at the time of disease presentation provides a new prognostic indicator for overall survival (Dearden et al., 2008). 
The aims of this study were two-fold; primarily to determine the prevalence of DAT positivity in newly diagnosed patients with CLL and secondarily to correlate DAT positivity with Rai staging, hematological parameters and biochemical markers. These outcomes are helpful for risk stratification, family counseling and to predict the overall survival as well as modifications in treatment strategies for better outcome.

\section{Materials and Methods}

This is a cross sectional prospective study conducted from January 2011 till June 2013.60 patients with chronic lymphoid leukemia were enrolled in the study. Sociodemographic data including age and gender were recorded. All patients underwent detailed history, general physical and systemic examination. Organomegaly was confirmed with abdominal ultrasound and computed tomography where indicated. Diagnosis of CLL was established by immunophenotyping either by immunohistochemistry or by flow cytometry technique.

Venous blood samples were taken for complete blood count, direct anti-globulin test, serum lactate dehydrogenase, serum urea, serum creatinine and uric acid. Hematological parameters were determined by Cell Dyne Ruby (Abott, Diagnostic). Biochemical markers were detected by Hitachi 912 (Japan) by photometric assay, while Direct coombs test was performed by column agglutination Gel technique (Diamed). The diagnosis of autoimmune hemolytic anemia (AIHA) was based on the presence of unexplained anemia and a positive DAT for $\mathrm{IgG}, \mathrm{C} 3 \mathrm{~d}$ or both prior to treatment or any blood product transfusion.

Statistical analysis: Statistical analysis was carried out using SPSS version 21. Qualitative data were presented in terms of frequencies and percentages. Mean and standard deviation were reported for quantitative variables. Independent sample t-test and chi-square test of independence were used to identify significant differences and associations between DAT and study variables.

\section{Results}

Sixty patients diagnosed with B-CLL were included in this study. $42(70 \%)$ were males and 18(30\%) were females. Male to female ratio were 2.1: 1. Mean age of our patients was $59 \pm 9.2$ years.

The direct anti-globulin test (polyspecific) positivity was found in 14 patients $(23.3 \%)$. The mono-specific IgG was positive in 11 patients $(18.3 \%), \mathrm{C} 3 \mathrm{~d}$ in 1 patient (1.6\%) and 2 patients (3.3\%) had both $\mathrm{IgG}$ and $\mathrm{C} 3 \mathrm{~d}$ positive. Mean hemoglobin was $10.8 \pm 2.4 \mathrm{gm} / \mathrm{dl}$, while other hematological and biochemical markers are shown in table-1.

Mean age of DAT negative and positive patients are statistically not significant $(\mathrm{P}>0.05)$. Low mean hemoglobin of $8.3 \pm 3.0 \mathrm{gm} / \mathrm{dl}$ was seen in DAT positive patients compared with DAT negative patients having mean hemoglobin level of $11.7 \pm 1.6 \mathrm{gm} / \mathrm{dl}(\mathrm{P}=0.001)$. However, DAT revealed positive association with advanced clinical RAI staging as positive DAT cases were

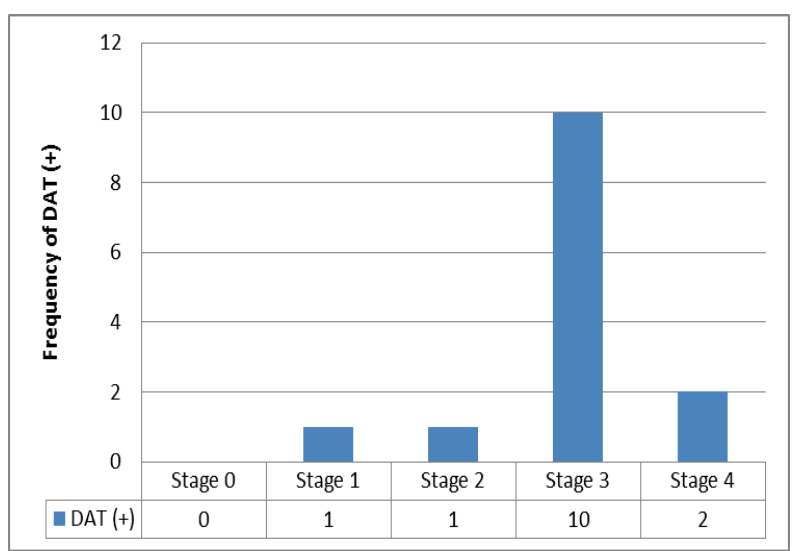

Figure 1. Prevalence of DAT (+) in Rai Staging

Table 1. Descriptive Statistics

\begin{tabular}{|c|c|c|}
\hline \multicolumn{2}{|l|}{ Parameters } & $\operatorname{Mean}(\mathrm{SD})$ \\
\hline \multicolumn{2}{|l|}{ Age } & $59.0(9.2)$ \\
\hline \multicolumn{2}{|l|}{ Hemoglobin } & $10.8(2.4)$ \\
\hline \multicolumn{2}{|l|}{ Total Leucocyte Count } & $91.5(87.8)$ \\
\hline \multicolumn{2}{|l|}{ Platelet Count } & $197.8(103.2)$ \\
\hline \multicolumn{2}{|l|}{ Absolute Lymphocyte Count } & $91.88(69.43)$ \\
\hline \multicolumn{2}{|l|}{ Serum urea } & 46.2(10.4) \\
\hline \multicolumn{2}{|l|}{ Serum creatinine } & $1.29(0.56)$ \\
\hline \multicolumn{2}{|l|}{ Serum Lactate dehydrogenase } & $512(232.61)$ \\
\hline \multicolumn{2}{|l|}{ Serum Uric Acid } & $5.59(2.47)$ \\
\hline & & Frequency $(\%)$ \\
\hline \multirow[t]{2}{*}{ Gender } & Male & $42(70 \%)$ \\
\hline & Female & $18(30 \%)$ \\
\hline DAT & Positive & $14(23.3 \%)$ \\
\hline (Polyspecific) & Negative & $46(76.7 \%)$ \\
\hline DAT & $\operatorname{IgG}$ & $11(18.3 \%)$ \\
\hline \multirow[t]{2}{*}{ (Monospecific) } & C3d & $1(1.6 \%)$ \\
\hline & $\operatorname{IgG} \& \mathrm{C} 3 \mathrm{~d}$ & $2(3.3 \%)$ \\
\hline \multirow[t]{5}{*}{ Rai-Staging } & Stage 0 & $7(11.6 \%)$ \\
\hline & Stage 1 & $8(13.3 \%$ \\
\hline & Stage 2 & $16(26.7 \%)$ \\
\hline & Stage 3 & $16(26.7 \%)$ \\
\hline & Stage 4 & $13(21.7 \%)$ \\
\hline
\end{tabular}

Table 2. Comparative Analysis of DAT Positive and Negative Patients

\begin{tabular}{lccc}
\hline Parameters & $\begin{array}{c}\text { DAT } \\
\text { Negative }\end{array}$ & $\begin{array}{c}\text { DAT } \\
\text { Positive }\end{array}$ & P-value \\
\hline Age & $58.1(9.9)$ & $61.1(6.4)$ & 0.3 \\
Hemoglobin & $11.7(1.6)$ & $8.3(3.0)$ & $0.001^{*}$ \\
Total Leucocyte Count & $94.3(94.5)$ & $82.7(62.5)$ & 0.6 \\
Platelet Count & $195.4(102.1)$ & $205.6(110.5)$ & 0.7 \\
Stage0 & $7(15.2 \%)$ & $0(0 \%)$ & $0.005^{*}$ \\
Stage I & $8(17.3 \%)$ & $1(7.1 \%)$ & \\
Stage II & $13(28.2 \%)$ & $1(7.1 \%)$ & \\
Stage III & $5(10.9 \%)$ & $10(71.4 \%)$ & \\
Stage IV & $13(28.2 \%)$ & $2(14.3 \%)$ & \\
\hline
\end{tabular}

more prevalent in Stage III disease which comprises of 10 patients $(\mathrm{P}=0.005)$. No associations were established with gender and biochemical markers.

\section{Discussion}

Autoimmune phenomena are a well known complication of lymphoproliferative neoplasm particularly 
of chronic lymphoid leukemia. Of all, AIHA is the most frequent autoimmune disorder depicted in CLL and on the contrary, CLL is the hematological malignancy in whom AIHA occurs most frequent. However, the exact mechanisms of autoimmune disturbance in CLL are still unclear.

Patients with anemia due to immune mechanisms have a better outcome than those in whom this features are due to bone marrow infiltration by the disease. This highlights the importance of determining the origin of the cytopenia in CLL patients for both prognostic and therapeutic reasons (Clive et al., 2010; Moreno et al., 2010).

Literature review illustrated that between $3 \%$ to as high as $37 \%$ of patients with CLL develop AIHA during the course of illness (Moreno et al., 2010).

It is already proven that development of AIHA has an important affiliation with positive DAT (Dearden et al., 2008). On the other hand it is also established in various studies that later or sooner most of the DAT positive CLL patients developed AIHA, particularly towards disease advancement.

We have described a series of 60 CLL cases followed at our institution including their clinical, hematological and biochemical profiles. Limited studies are available from Asian countries. Previously, a single study conducted in northern part of Pakistan has reported $19.4 \%$ of CLL patients showing DAT positivity and its association with significant anemia (Ehsan et al., 2013). This finding is comparable to that of our study. When compared with our neighbor India, one study reported low prevalence of DAT positivity accountable in only $3.1 \%$ of CLL patients having immune mediated hemolysis (Agrawal et al., 2007).

Various studies conducted in United State, Israel, Spain, England and Rome have reported a frequency of positive DAT in $4.5 \%, 7 \%, 4.3 \%, 7.7 \%$ and $3.7 \%$ of CLL patients respectively (Kyasa et al., 2003; Lischner et al., 1988; Moreno et al., 2010; Hamblin et al., 1986; Mauro et al., 2000). However the prevalence of DAT positivity in our patients appears to be higher than these studies conducted in western world. This difference may be accredited to the aggressive advanced disease course along with concomitant factor of delay in seeking medical attention. This might also be due to more immune disturbance in our setting.

A multicenter Gimema study showed that $6.5 \%$ of the total patients with CLL had developed autoimmune cytopenias in which $66 \%$ of patients had developed AIHA (Barcellini et al., 2006). 89\% of these were having isolated IgG positivity while remaining $11 \%$ were $\mathrm{C} 3 \mathrm{~d}$ positive (Barcellini et al., 2006). Similarly our DAT positive patients are predominantly of IgG type which is seen in 11 out of 14 patients $(78.6 \%)$.

The National Cancer Institute Sponsored Working Group Guidelines for CLL included AIHA among CLL as signs of active disease (Hallek et al., 2008). Auto immune hemolytic anemia is frequently associated with advance stage of CLL. It has been used as a surrogate marker and has a significant effect on overall survival. (Mauro et al., 2000; Dearden et al., 2008).

The relationship between AIHA and advanced clinical stage has been a consistent finding across different studies.
It was reported that AIHA occurring in $11 \%$ of advanced stage CLL patients (Diehl et al., 1998). Another study conducted in England detected that DAT positivity is less prevalent in stage A disease accountable in $2.9 \%$ of patients compared to $13.3 \%$ in advanced stage disease (Hamblin et al., 1986). Dearden et al concluded that DATpositivity at the time of diagnosis was associated with more advanced stage of disease (Dearden et al, 2008). The findings of present study are parallel to previous studies; as out of 14 patients $(23.3 \%)$ with DAT positivity, 10 patients $(16.6 \%)$ were in advanced Rai stage III disease.

The development of AIHA were associated with a poor overall survival, it was proved that DAT could be a sovereign prognostic marker or at least surrogate marker when associated with other poor prognostic markers such as increase in immunoglobulin heavy chain mutation status (Dearden et al., 2008). Later on supported by another study that a high level of $\beta 2$-microglobulin and unmutated IgHV are often related to DAT positivity (Ricci et al., 2013). Thus, a laboratory test for DAT could be an important adjunct in the patients overall management.

The results of present study, conducted in southern part of country shows a significant difference in prevalence of DAT positivity when compared with international data; however it is comparable to locally published work. Thus the geographical variations, genetic makeup and natural history of the disease in both parts of the region could be the contributory factors of high prevalence in our part.

The limitations of the study need to be mentioned; one is small sample size in our study. A large sample would be a better indicator of DAT prevalence in our population and secondly is the lack of follow up details to have association with clinical outcome in these patients. Despite the limitations mentioned above, strength of this study is the fact that this is first local study which provides essential local informative data for prognostic and therapeutic stratification in our setup.

In conclusion, Unlike the western studies, DAT positivity appears relatively high; indicating predominance of immune disturbances in our setting. Significant association of positive DAT was found with higher degree of AIHA and advanced Rai stage. DAT positivity can be considered as a surrogative marker for advanced clinical disease in these patients. Further prospective studies on large sample size should be accompanied to interogate association of this emerging marker with other prognostic markers.

\section{Acknowledgements}

The authors are grateful to the patients who have participated in this study. We thank Mr Abrar Hussain of the Hematology Division of Liaquat National Hospital for his excellent support.

\section{References}

Agrawal N, Naithani R, Mahapatra M, et al (2007).Chronic lymphocytic leukemia in India-a clinico-hematological profile. Hematology, 12, 229-33.

Barcellini W, Capalbo S, Agostinelli RM, et al (2006). 
Syeda Alia Abbas et al

Relationship between autoimmune phenomena and disease stage and therapy in B-cell chronic lymphocytic leukemia. Haematologica, 91, 1689-92.

Clive S. Zent, MD, Neil E, Kay (2010). Autoimmune complications in chronic lymphocytic leukemia. Best Pract Res Clin Haematol, 23, 47-59.

De Rossi G, Granati L, Girelli G, et al (1988). Incidence and prognostic significance of autoantibodies against erythrocytes and platelets in chronic lymphocytic leukemia. Nouvelle Revue Francaise D'hematologie, 30, 403-6.

Dearden C, Wade R, Else M, et al, (2008). The prognostic significance of a positive direct anti-globulin test in chronic lymphocytic leukemia: a beneficial effect of the combination of fludarabine and cyclophosphamide on the incidence of hemolytic anemia. Blood, 111, 1820-6.

Diehl LF, Ketchum LH (1998). Autoimmune disease and chronic lymphocytic leukemia: autoimmune hemolytic anemia, pure red cell aplasia and autoimmune thrombocytopenia. Semin Oncol, 25, 80.

Ehsan A, Lone A, Khan MA (2013). Autoimmune complications in chronic lymphocytic leukemia: a single center experience. Biomedica, 9, 37-41.

Engelfriet CP, Overbeeke MAM, Von dem Borne AEGKR (1992). Autoimmune hemolytic anemia. Semin Hematol, 29, 3 .

Foon KA, Rai KR, Gale RP (1990). Chronic lymphocytic leukemia: new insights into biology and therapy. Ann Intern Med, 113, 525-39.

Hamblin TJ, Oscier DG, Young BJ (1986). Autoimmunity in chronic lymphocytic leukaemia. J Clin Pathol, 39, 713-6.

Hallek M, Cheson BD, Catovsky D, et al (2008). International Workshop on Chronic Lymphocytic Leukemia. Guidelines for the diagnosis and treatment of chronic lymphocytic leukemia: a report from the International Workshop on Chronic Lymphocytic Leukemia updating the National Cancer Institute-Working Group 1996 guidelines. Blood, 111, 5446-56.

Hodgson K, Ferrer G, Montserrat E, Moreno C (2011). Chronic lymphocytic leukemia and autoimmunity: a systematic review. Haematologica, 96, 752-61.

Kermani IA, Dehdilani M, Dolatkhah R (2007). Chronic lymphocytic leukemia in the recent 10 years and treatment effects of Fludarabin. Asian Pac J Cancer Prev, 8, 367-71.

Kyasa MJ, Parrish RS, Schichman SA, et al (2003). Autoimmune cytopenia does not predict poor prognosis in chronic lymphocytic leukemia/small lymphocytic lymphoma. Am J Hematol, 74, 1-8.

Lischner M, Prokocimer M, Zolberg A, et al (1988). Autoimmunity in chronic lymphocytic leukaemia. Postgrad Med J, 64, 590-2.

Mauro FR, Foa R, Giannarelli D, et al (1999). Clinical characteristics and outcome of young chronic lymphocytic leukemia patients: a single institution study of 204 cases. Blood, 94, 448-54.

Mauro FR, Foa R, Cerretti R, et al (2000). Autoimmune hemolytic anemia in chronic lymphocytic leukemia: clinical, therapeutic, and prognostic features. Blood, 95, 2786-92.

Moreno C, Hodgson K, Ferrer G, et al (2010). Autoimmune cytopenia in chronic lymphocytic leukemia: prevalence, clinical associations, and prognostic significance. Blood, 116, 4771-6.

Moreno C, Montserrat E (2008). New prognostic markers in chronic lymphocytic leukemia. Blood, 22, 211-9.

Mozaheb Z, Hasanzadeh N, Abadi MH, Aghaee MA (2012). Chronic lymphocytic leukemia and prognostic factors. Asian Pac J Cancer Prev, 13, 3009-13.

Polliack A, Lugassy C (1992). Autoimmunity and auto-immune syndromes associated with and preceding the development of lymphoproliferative disorders. Leukemia, 6, 152.

Ricci F, Tedeschi A, Vismara E, et al (2013). Should a positive direct antiglobulin test be considered a prognostic predictor in chronic lymphocytic leukemia? Clin Lymphoma Myeloma Leuk, 13, 441-6.

Rozman C, Montserrat E (1995). Chronic lymphocytic leukemia. N Engl J Med, 1333, 1052-7.

Shahjahani M , Mohammadiasl J, Noroozi F, et al (2015). Molecular basis of chronic lymphocytic leukemia diagnosis and prognosis. Cell Oncol Dordr, 38, 93-109.

$\mathrm{Xu}$ W, Li JY, Miao KR, et al (2009). The negative prognostic significance of positive direct antiglobulin test in Chinese patients with chronic lymphocytic leukemia. Leuk Lymphom, 50, 1482-7.

Zent CS, Ding W, Schwager SM, et al (2008). The prognostic significance of cytopenia in chronic lymphocytic leukaemia/ small lymphocytic lymphoma. Br J Haematol, 141, 615-21.

Zeeshan R, Sultan S, Irfan SM, Kakar J, Hameed MA (2015). Clinico-hematological profile of patients with B-chronic lymphoid leukemia in Pakistan. Asian Pac J Cancer Prep, 16, 793-6.

Zeeshan R, Irfan SM, Sultan S, Bhimani S (2015). ZAP-70 protein expression in B-cell Chronic Lymphoid Leukemia: A single center experience from Pakistan. Asian Pac J Cancer Prev, 16, 1587-90.

Zwiebel JA, Cheson BD (1998). Chronic lymphocytic leukemia: staging and prognostic factors. Semin Oncol, 25, 42-59. 